\title{
A Randomized, Placebo-controlled Trial of Digestive Enzymes in Children with Autism Spectrum Disorders
}

\author{
Khaled Saad', Azza A. Eltayeb', Ismail L. Mohamad', Abdulrahman A. Al-Atram², Yasser Elserogy ${ }^{3}$, \\ Geir Bjørklund ${ }^{4}$, Amira A. El-Houfey ${ }^{5,6}$, Bubba Nicholson ${ }^{7}$ \\ 'Department of Pediatrics, Faculty of Medicine, Assiut University, Assiut, Egypt, ${ }^{2}$ Department of Psychiatry, College of Medicine, Almajmaah \\ University, Kingdom of Saudi Arabia, ${ }^{3}$ Department of Neuropsychiatry, Assiut University, Assiut, Egypt, ${ }^{4}$ Council for Nutritional and \\ Environmental Medicine, Mo i Rana, Norway, ${ }^{5}$ Department of Community Health Nursing, Faculty of Nursing and Health Allied, Jazan \\ University, Kingdom of Saudi Arabia, ${ }^{6}$ Faculty of Nursing, Assiut University, Assiut, Egypt, ${ }^{7}$ Nicholson Science, Tampa, FL, USA
}

\begin{abstract}
Objective: There is growing evidence for a gut-brain connection associated with autism spectrum disorders (ASDs). This suggests a potential benefit from introduced digestive enzymes for children with ASD.

Methods: We performed a double-blind, randomized clinical trial on 101 children with ASD (82 boys and 19 girls) aged from 3 to 9 years. ASD patients were diagnosed according to the Diagnostic and Statistical Manual of Mental Disorders 4th edition, text revision (DSM-IV-TR) diagnostic criteria. Structured interviews of at least one hour each both with the parents and the child were performed. Later on, another two hours-session was conducted applying the Childhood Autism Rating Scale (CARS). ASD patients were randomized to receive digestive enzymes or placebo.

Results: The ASD group receiving digestive enzyme therapy for 3 months had significant improvement in emotional response, general impression autistic score, general behavior and gastrointestinal symptoms. Our study demonstrated the usefulness of digestive enzyme in our population of ASD patients.

Conclusion: Digestive enzymes are inexpensive, readily available, have an excellent safety profile, and have mildly beneficial effects in ASD patients. Depending on the parameter measured in our study, we propose digestive enzymes for managing symptoms of ASD. Digestive enzyme therapy may be a possible option in treatment protocols for ASD in the future.
\end{abstract}

KEY WORDS: Autism spectrum disorders; Digestive enzymes; Child; Clinical trial.

\section{INTRODUCTION}

Autism is a behaviorally defined syndrome beginning before three years of age characterized by pervasive deficits in social interaction, impairment in verbal and nonverbal communication, and stereotyped patterns of interests and activities. Currently there is no curative treatment for autism. Management includes education, supportive and behavioral management. Although pharmacological therapies provide an adjunct to behavioral interventions, they have no effect on changing the core symptoms. ${ }^{1,2)}$ The etiology of autism remains unclear, although several theories have been put forward. ${ }^{1)}$

\footnotetext{
Received: December 8, 2014 / Revised: January 30, 2015

Accepted: March 4, 2015

Address for correspondence: Khaled Saad, MD

Department of Pediatrics, Faculty of Medicine, Assiut University, Assiut 71516, Egypt

Tel: +20-100-608-0182, Fax: +20-88-236-8371

E-mail: khaled.ali@med.au.edu.eg
}

Gastrointestinal disorders are among the medical pathologies associated with autism. The association of gastrointestinal disorders and autism is well known, with symptom occurrence ranging from $9 \%$ to $70 \%$ or higher. ${ }^{3,4)}$ The gastrointestinal symptoms most frequently identified in connection with autism are constipation, diarrhea, bloating, belching, abdominal pain, reflux, vomiting, and flatulence. Various hypotheses have been formulated since the 1990s on the etiopathogenetic role played by the interaction between the gut and the brain in causing autistic behaviors. ${ }^{3,4)}$ At present, there are no confirmed data on the relation between gastrointestinal disorders and autism spectrum disorders (ASDs), and it is possible that different mechanisms and different genes are implicated in a group of subjects with ASDs and gastrointestinal and immune system disorders. Variables considered in the gut-brain axis model include bowel inflammatory diseases, food allergies, increased intestinal permeability, the interaction between the immunological system and the

(a) This is an Open-Access article distributed under the terms of the Creative Commons Attribution Non-Commercial License (http://creativecommons.org/licenses/by-nc/4.0) which permits unrestricted non-commercial use, distribution, and reproduction in any medium, provided the original work is properly cited. 
blood-brain barrier, microglia, astrocytes, and neuronal modulation; however, more studies are necessary to clarify this relationship. ${ }^{3-5)}$ Exorphins are compounds, formed outside of the brain, that interact with opiate receptors; especially any of a range of soluble proteins. These exorphins (i.e., casomorphins and gluteomorphins or gliadorphin) are then easily transferred across the lumen of the gut into the circulation where they exert opioid-type action on the brain., ${ }^{3,6)}$ There is growing evidence for a gut-brain connection associated with ASD. This tenuous correlation does suggest a plausible benefit from digestive enzymes with meals to aid digestion of all exorphin peptides and disaccharides, especially for ASD children with gastrointestinal disturbance. Enzyme therapy has recently been employed to try to remove exorphins from the system. This has been based on supplementation with large amounts of proteases from the different categories of proteolytic enzymes and these have included acid or carboxyl peptidases, serine, cysteine and zinc proteases. Additionally, peptidases with both endo- and exo-peptidase activity have been favored. One approach has been to use a large amount of peptidase to specifically digest the exorphins. While sound in theory, it has only met with limited success. The data below address the question of whether or not a more proactive enzyme formulation can have a positive effect on gut- brain parameters in ASD. ${ }^{3,6)}$ Accordingly theories suggest that digestion products of natural foods such as those containing gluten or casein are able to enter the blood through the 'leaky' mucosa and induce antigenic responses and directly affect the central nervous system will be beneficial to control gastrointestinal troubles among ASD patients. There are limited data on the efficacy of digestive enzymes in children with ASD, only 2 studies; the first one was a case series ${ }^{6)}$ and the second was a double-blind placebo-controlled trial, ${ }^{1)}$ which will be discussed later. Therefore, this study aims to evaluate the efficacy of digestive enzymes in an Egyptian cohort of children with ASD.

\section{METHODS}

The Ethical Committee of Assiut University, Assiut, Egypt, approved the study. An informed written consent in accordance with Assiut University Ethical Committee guidelines was taken from guardians of all cases and controls. This was a three months, double blinded, randomized, placebo-controlled trial undertaken in the outpatient clinic for children at Assiut University hospitals and 2 private centers in Assiut city, Egypt.

\section{Patients}

Patients included 101 outpatients ( 82 boys and 19 girls) between the ages of 3 and 9 years. All patients were recruited from the neuropediatric clinics Assiut university hospitals and 2 private centers for ASD in Assiut city, Upper Egypt. A total of 235 patients were screened for eligibility, and 101 were enrolled (32 failed to provide consent, and 102 did not meet eligibility criteria and/or one or more exclusion criteria). The diagnosis of ASD was established by a senior child psychiatrist before participants were initiated into the study. Also, he performed all neuropsychiatric assessments and followed up the cases.

Exclusion criteria: Children with a history of other developmental disorders or psychiatric diseases were excluded. In addition, patients were excluded if they had a clinically significant chronic medical condition, including; anemia, brain malformations, metabolic diseases, seizures, and current use of pharmacotherapy (e.g., psychiatric medications) within the preceding 6 months. Also, patients with gastrointestinal disease associated with malabsorption were excluded. Finally, all patients were not started on any other therapies as vitamins or other alternative therapies for 2 weeks before and during the study period.

\section{Methodology}

Investigated patients were evaluated according to the following

Detailed history (carefully gathered from parent interview, paying attention to family history of consanguinity, similar conditions of ASD), social activities, self-care, and time of onset of ASD. Meticulous neurological examinations (including sensory, motor, and autonomic evaluations) were done for all patients. Hematology tests and stool analysis were collected at baseline for exclusion of anemia and parasitic infestations; physical examinations and vital signs were evaluated at baseline and at the end of the study.

Autism spectrum disorders were diagnosed according to the Diagnostic and Statistical Manual of Mental Disorders 4th edition, text revision (DSM-IV-TR) diagnostic criteria. ${ }^{7}$ Structured interviews of at least 2 hours, each both with the parents and the child were performed, in a room equipped with play material appropriate for age level. Later on, another 2-3 hours-session was conducted for the assessment of ASD severity and follow up the patients utilizing the Childhood Autism Rating Scale $(\mathrm{CARS})^{8)}$ and Global Behavior Rating Scales (GBRS). ${ }^{9)}$ 
Parents were carefully asked to rate the severity of the ASD symptoms that their children displayed at home by using the previous scales. CARS is a well-established scale for the screening and classification of childhood autism with good agreement between DSM-IV-TR diagnostic criteria and CARS. ${ }^{8)}$ The scale assesses behavior in 14 domains that are affected by severe problems in ASD, plus one general category of impressions of ASD, with the aim of identifying children with ASD, as differentiated from the other developmental disorders. The examiner assigned a score of 1 to 4 for each item: 1 indicates behavior appropriate for age level, while 4 indicates a severe deviance with respect to the normal behavior for age level. The scores for the single items are added together into a total score, which classifies the child as not autistic (below 30), mild or moderately autistic (30-36.5) or severely autistic (above 36.5).

In a double-blind, randomized clinical trial; patients were randomized to receive digestive enzymes (group 1) or placebo (group 2) by using a random number generator. Both digestive enzymes and placebo were in syrup form and were identical in shape with nearly the same taste and color (no differences were detected). Assignments were kept in sealed envelopes until data analysis. Randomization and allocations were blind. Group 1 consisted of 51 patients (41 males) were randomly allocated to receive the digestive enzyme syrup; Neo-Digestin oral solution. Each $100 \mathrm{ml}$ of the solution contains: Papain $1.6 \mathrm{~g}$ and pepsin $0.8 \mathrm{~g}$. Papain belongs to a family of related proteins with a wide variety of activities, including endopeptidases, aminopeptidases, dipeptidyl-peptidases and enzymes with both exo- and endo-peptidase activity, the combination of papain and pepsin catalyze the breakdown of proteins. ${ }^{10)}$ According to the manufacturer the patients received $15 \mathrm{ml} /$ day $(5 \mathrm{ml}$ with each meal, usually at the start of the meal). Group 2 consisted of 50 patients (41 males) received a placebo solution (sucralose syrup $25 \%$ ) with identical packaging as group 1, for 3 months. Throughout the study, the patients who administered their own medications and their parents were blind to assignments. The measures for the outcome of the therapy were the CARS and GBRS. The GBRS ${ }^{9)}$ provides a seven point scale to rate the child's behavior over a period of time in comparison to his/her condition before the initial observations. The scale parameters for general behavior were defined as follows: 1 , much worse; 2 , moderately worse; 3 , little worse; 4 , no change; 5 , little better; 6 , moderately better; and 7 , much better. In addition to this behavior rating, parents were asked to rate their child in five additional cate- gories of gastrointestinal symptoms (quality of stools, abdominal pain, vomiting, food variety), nighttime sleeping, daytime wetting, and nighttime wetting. ${ }^{9)}$

All patients were assessed by a child psychiatrist at the beginning, and 3 months after the medication started. All patients were followed up every 2 weeks for treatment compliance and any addition or changes of the medications. Side effects were recorded throughout the study and were assessed using a checklist at 1,2, and 3 months.

\section{Statistical Analysis}

Statistical Package for Social Sciences (SPSS) program version 16 (SPSS Inc., Chicago, IL, USA) was used for data analysis. Descriptive statistics as minimum, maximum and mean \pm standard deviation and independent sample $t$-test, correlation and linear regression. $p$ value of $<0.05$ denoted the presence of a statistically significant difference.

\section{RESULTS}

No significant difference was identified between the two groups as regard demographic data including age, sex, weight and classification of autism (Table 1). Before the beginning of the study, 4 patients had mild gastrointestinal manifestation; one had constipation and 3 had recurrent diarrhea. There was no significant difference between patients with gastrointestinal problems and other children with ASD.

A total of 92 children (47 in group 1 and 45 in group 2) completed the study fully over the 3 -months period. Four patients were excluded from the group 1 due to side effects of the therapy in two patients and refusal of syrup in another two. Five patients excluded from the group 2 due to refusal of syrup by children. The side effects noted by the research team during the 3-months study period included

Table 1. The demographic data and classification of autism of the studied groups

\begin{tabular}{lccc}
\hline \multicolumn{1}{c}{ Item } & $\begin{array}{c}\text { Group 1 } \\
(\mathrm{n}=47)\end{array}$ & $\begin{array}{c}\text { Group 2 } \\
(\mathrm{n}=45)\end{array}$ & p value \\
\hline Age (yr) & $5.94 \pm 2.01(3-9)$ & $5.87 \pm 2.12(3-9)$ & NS \\
Sex (male : female) & $39: 8$ & $38: 7$ & NS \\
Weight (kg) & $19.89 \pm 8.31$ & $20.02 \pm 7.22$ & NS \\
Severe autism & 9 & 8 & NS \\
Mild/moderate autism & 37 & 37 & NS \\
\hline
\end{tabular}

Values are presented as mean \pm standard deviation (range) or number only.

Group 1, group received digestive enzymes; group 2, placebo group.

NS, non-significant. 
skin rashes, itching and abdominal pain. All were mild, transient and only two patients discontinued the treatment.

There was no significant difference between both groups in CARS scores before intervention (Table 2). After the study 3-months duration, the CARS scores were significantly different between both groups in 2 parameters; the emotional response score $(p=0.027)$, and general autistic impression score $(p=0.042)$ which were significantly improved in patients of group 1 receiving digestive enzymes (Table 2). In addition to a significant improvement in total CARS scores 3-8 points. As regards GBRS outcome results, children in group 1 with digestive enzyme therapy had significant improvement in two parameters including general behavior and gastrointestinal symptoms (quality of stools, abdominal pain, vomiting and food variety) ( $p \leq 0.001$ for each) (Table 3 ). The main behavioural changes in our patients after treatment were improvement in restricted repetitive behaviors and stereotypic behaviors. There was no significant correlation between improvement of gastrointestinal manifestation and CARS scores. So, the behavioural improvement in ASD patients could be attributed to the digestive enzymes directly, in addition to the relief of gastrointestinal symptoms. There was no significant difference between the two groups in daytime wetting, nighttime wetting and nighttime sleeping.

\section{DISCUSSION}

There are numerous reports of gastrointestinal dis-

Table 2. CARS scores in enzyme and placebo groups before and after treatment

\begin{tabular}{|c|c|c|c|c|c|}
\hline \multirow{2}{*}{ Outcome measure } & \multicolumn{2}{|c|}{ Group 1} & \multicolumn{2}{|c|}{ Group 2} & \multirow{2}{*}{ Significance } \\
\hline & Before enzyme therapy & After enzyme therapy & Before placebo & After placebo & \\
\hline Relating to People score & $2.02 \pm 1.4$ & $2.17 \pm 1.0$ & $2.20 \pm 1.9$ & $2.35 \pm 1.8$ & $p^{*+\dagger}=\mathrm{NS}$ \\
\hline Emotional Response score & $2.30 \pm 1.6$ & $1.02 \pm 0.7$ & $2.00 \pm 1.3$ & $1.98 \pm 1.4$ & $p^{*}=\mathrm{NS}, p^{\dagger}=0.027$ \\
\hline Imitation score & $2.56 \pm 1.4$ & $3.01 \pm 1.0$ & $2.71 \pm 1.2$ & $2.96 \pm 0.9$ & $p^{* \dagger}=\mathrm{NS}$ \\
\hline Body Use score & $2.90 \pm 2.1$ & $3.07 \pm 1.7$ & $2.88 \pm 1.6$ & $3.02 \pm 1.5$ & $\mathrm{P}^{* \cdot \dagger}=\mathrm{NS}$ \\
\hline Object Use score & $1.22 \pm 1.1$ & $1.03 \pm 0.8$ & $1.19 \pm 0.8$ & $0.99 \pm 1.0$ & $\mathrm{p}^{*, \dagger}=\mathrm{NS}$ \\
\hline Adaptation to Change score & $2.03 \pm 0.4$ & $1.73 \pm 0.9$ & $2.19 \pm 0.5$ & $1.81 \pm 0.7$ & $\mathrm{P}^{* \cdot \dagger}=\mathrm{NS}$ \\
\hline Listening Response score & $1.90 \pm 1.1$ & $1.51 \pm 1.2$ & $2.01 \pm 0.8$ & $1.48 \pm 0.5$ & $\mathrm{p}^{* \cdot \dagger}=\mathrm{NS}$ \\
\hline Taste, Smell, Touch score & $1.50 \pm 1.3$ & $2.02 \pm 1.1$ & $1.61 \pm 1.0$ & $2.10 \pm 0.9$ & $\mathrm{P}^{*}{ }^{\dagger}=\mathrm{NS}$ \\
\hline Visual Response score & $2.59 \pm 1.5$ & $2.19 \pm 1.3$ & $2.71 \pm 1.1$ & $2.04 \pm 1.3$ & $\mathrm{p}^{*}{ }^{\dagger}=\mathrm{NS}$ \\
\hline Fear or Nervous score & $2.50 \pm 0.8$ & $2.45 \pm 0.7$ & $2.67 \pm 0.7$ & $2.77 \pm 1.0$ & $p^{*, \dagger}=\mathrm{NS}$ \\
\hline Verbal Communication score & $3.11 \pm 1.2$ & $2.98 \pm 1.2$ & $2.91 \pm 1.4$ & $3.02 \pm 1.2$ & $\mathrm{p}^{*, \dagger}=\mathrm{NS}$ \\
\hline Activity Level score & $1.99 \pm 0.7$ & $2.01 \pm 0.8$ & $2.09 \pm 0.7$ & $2.00 \pm 0.6$ & $\mathrm{P}^{* \cdot \dagger}=\mathrm{NS}$ \\
\hline Nonverbal Communication score & $3.12 \pm 1.3$ & $3.07 \pm 1.7$ & $3.28 \pm 1.9$ & $3.20 \pm 1.5$ & $\mathrm{P}^{* \cdot \dagger}=\mathrm{NS}$ \\
\hline $\begin{array}{l}\text { Level \& Consistency of } \\
\text { Intellectual Response score }\end{array}$ & $2.57 \pm 1.0$ & $2.60 \pm 1.2$ & $2.41 \pm 1.6$ & $2.70 \pm 1.3$ & $p^{*, \dagger}=N S$ \\
\hline General Impression score & $2.92 \pm 1.5$ & $1.52 \pm 0.6$ & $3.07 \pm 1.1$ & $2.47 \pm 1.5$ & $p^{*}=\mathrm{NS}, p^{\dagger}=0.042$ \\
\hline Total CARS score & $36.1 \pm 3.7$ & $31.2 \pm 1.2$ & $35.3 \pm 4.0$ & $35.5 \pm 2.8$ & $p^{*}=\mathrm{NS}, p^{\dagger}=0.034$ \\
\hline
\end{tabular}

Values are presented as mean \pm standard deviation.

CARS, Childhood Autism Rating Scale.

Group 1, group received digestive enzymes; group 2, placebo group.

$p^{*}$, comparison between enzyme and placebo groups before treatment; $p^{\dagger}$, comparison between enzyme and placebo groups after treatment; NS, non-significant ( $p \leq 0.05$ : significant).

Table 3. GBRS outcome mean scores in placebo and enzyme groups after treatment

\begin{tabular}{|c|c|c|c|c|c|}
\hline \multirow{2}{*}{ Outcome measure } & \multicolumn{2}{|c|}{ Group 1} & \multicolumn{2}{|c|}{ Group 2} & \multirow{2}{*}{ Significance } \\
\hline & Before enzyme therapy & After enzyme therapy & Before placebo & After placebo & \\
\hline Child's general behavior & $3.01 \pm 1.3$ & $5.5 \pm 0.75$ & $3.50 \pm 0.7$ & $3.39 \pm 1.01$ & $p^{*}=\mathrm{NS}, p^{\dagger}<0.001$ \\
\hline Nighttime sleeping & $3.89 \pm 1.3$ & $4.02 \pm 1.20$ & $3.84 \pm 1.0$ & $3.98 \pm 0.89$ & $p^{*, \dagger}=N S$ \\
\hline Gastrointestinal symptoms $^{\dagger}$ & $3.79 \pm 0.61$ & $6.03 \pm 0.56$ & $4.02 \pm 0.28$ & $4.02 \pm 0.23$ & $p^{*}=\mathrm{NS}, p^{\dagger}<0.001$ \\
\hline Daytime wetting & $3.86 \pm 0.67$ & $3.97 \pm 0.88$ & $3.92 \pm 0.83$ & $4.03 \pm 1.04$ & $p^{*, \dagger}=\mathrm{NS}$ \\
\hline Nighttime wetting & $4.60 \pm 0.97$ & $4.23 \pm 1.25$ & $3.92 \pm 0.6$ & $4.09 \pm 0.64$ & $p^{*, \dagger}=N S$ \\
\hline
\end{tabular}

Values are presented as mean \pm standard deviation.

GBRS, Global Behavior Rating Scales.

Group 1, group received digestive enzymes; group 2, placebo group.

$p^{*}$, comparison between enzyme and placebo groups before treatment; $p^{\dagger}$, comparison between enzyme and placebo groups after treatment; NS, non-significant ( $p \leq 0.05$ : significant).

${ }^{\dagger}$ Quality of stools, abdominal pain, vomiting, food variety. 
turbances in children with ASD. Most of these studies reported that chronic gastrointestinal problems are common in autistic children and may complicate clinical management, can contribute to behavioral impairment, and should be evaluated and treated. ${ }^{3-5,11,12)}$ Lack of digestive enzymes in autistic patients was reported by many researchers. ${ }^{13-15)}$ They reported that children with ASD and gastrointestinal problems have low levels of digestive enzymes, especially lactase. Insufficient lactase may contribute to abdominal discomfort, pain and observed aberrant behavior. Most autistic children with lactose intolerance are not identified by clinical evaluation. ${ }^{11,13-15)}$

Horvath et al. ${ }^{13)}$ evaluated disaccharidase activity from endoscopic biopsies in 36 children with ASD. They found decreased activity of one or more disaccharidases or glucoamylase in $21(58.3 \%)$ of children. The most frequent finding was a low lactase level, which was present in 14 of 36 patients. All of the 21 children with low enzyme activities had loose stools and/or gaseousness. A large study ${ }^{15}$ involving intestinal biopsy samples of 199 individuals with ASD to determine the frequency of enzyme deficiency. They found that $58 \%$ of autistic children $\leq 5$ years of age were lactase deficient; whereas the older children with ASD, $65 \%$ were lactase deficient. In addition, $6 \%$ of ASD patients had mucosal inflammation. These problems seemed to be common in children and adults, suggesting that these problems are lifelong. ${ }^{15)}$

In our double-blind placebo controlled trial of digestive enzymes in ASD children, the group which received digestive enzyme therapy for 3 months had significant improvement in 4 parameters according to CARS and GBRS. The parents of this group rated significantly improved emotional response, general impression score, general behavior and gastrointestinal symptoms (quality of stools, abdominal pain, vomiting and food variety). From our point of view, these parameters are clinically important and encouraging to use digestive enzymes to ASD children. In agreement of our results, Brudnak et al. ${ }^{6}$ performed a case series study with post-test outcomes following open-label treatment with a 12 -week trial of digestive enzymes in children with ASD. They used digestive enzymes for proteins, peptides, casein, and phytic acid. Twenty-nine of the 46 subjects completed the trial, and 17 dropped out due to lack of palatability and behavioral or medical side effects. Of the 29 who completed the study, all reported improvements in many areas, especially socialization and hyperactivity and $50 \%$ of patients reported improvements in digestion. The drawback of this study was its open, uncontrolled design; however, the authors ${ }^{6}$ reported a significant positive trend for each of the $13 \mathrm{pa}-$ rameters measured on the symptom outcome survey.

Contrary, to the results of our study and the previous study, ${ }^{6}$ there was a more rigorous randomized, double-blind, placebo-controlled, crossover study ${ }^{1)}$ lasting 6 months and involving 43 participants aged 3-8 years. It involved a proteolytic enzyme supplement on the behavior, expressive language and other symptoms of a cohort of children diagnosed with ASD. The digestive enzymes were well tolerated, but there were no statistically significant clinical improvements on any parameter except food variety score, which was the only outcome measure that showed a statistically significant improvement on enzyme therapy compared with placebo. ${ }^{1)}$

Digestive enzymes are inexpensive, readily available, have an excellent safety profile, and have mildly beneficial effects in ASD patients. Depending on the parameter measured in our study; we propose digestive enzymes for managing symptoms of ASD. Digestive enzyme therapy may be a possible option in treatment protocols for ASD in the future. The primary limitation of our study was the small sample size; so more randomized controlled trials using a larger number of ASD patients with long-term follow-up are urgently needed in the near future.

There are several studies of gastrointestinal disturbances in autistic children, and most of these studies reported that these problems may contribute to behavioral impairment. Lack of digestive enzymes, especially lactase in autistic children was previously reported. Insufficient lactase may contribute to abdominal discomfort, pain, and observed aberrant behavior. Most autistic children with lactose intolerance are not identified by clinical evaluation.

In our double-blind placebo controlled trial of digestive enzymes in children with ASD (3-9 years), the group which received digestive enzyme therapy for 3 months had significant improvement in emotional response, general behavior, and gastrointestinal symptoms (quality of stools, abdominal pain, vomiting and food variety). From our point of view, these parameters are clinically important and encourage the use of digestive enzymes which are inexpensive, readily available and have an excellent safety profile - to children with ASD.

\section{REFERENCES}

1. Munasinghe SA, Oliff C, Finn J, Wray JA. Digestive enzyme supplementation for autism spectrum disorders: a doubleblind randomized controlled trial. J Autism Dev Disord 2010:40:1131-1138.

2. Saad K, Hammad E, Abdel-rahman AA, Sobhy KM. Autistic symptoms in late diagnosed phenylketonuric children in 
upper Egypt. J Neurol Res 2013;3:51-55.

3. Parmeggiani A. Gastrointestinal disorders and autism. In: Patel VB, Preedy VR, Martin CR, editors. Comprehensive guide to autism. New York:Springer Science plus Business Media;2014. p.2035-2046.

4. Adams JB, Audhya T, McDonough-Means S, Rubin RA, Quig D, Geis E, et al. Effect of a vitamin/mineral supplement on children and adults with autism. BMC Pediatr 2011;11:111.

5. Lofthouse N, Hendren R, Hurt E, Arnold LE, Butter E. A review of complementary and alternative treatments for autism spectrum disorders. Autism Res Treat 2012;2012: 870391.

6. Brudnak MA, Rimland B, Kerry RE, Dailey M, Taylor R, Stayton B, et al. Enzyme-based therapy for autism spectrum disorders-is it worth another look? Med Hypotheses 2002; 58:422-428.

7. American Psychiatric Association. Diagnostic and statistical manual of mental disorders: DSM-IV-TR. Washington, DC:American Psychiatric Association;2000.

8. Rellini E, Tortolani D, Trillo S, Carbone S, Montecchi F. Childhood Autism Rating Scale (CARS) and Autism Behavior Checklist $(A B C)$ correspondence and conflicts with DSM-IV criteria in diagnosis of autism. J Autism Dev Disord 2004;34:703-708.
9. Levy SE, Souders MC, Wray J, Jawad AF, Gallagher PR, Coplan J, et al. Children with autistic spectrum disorders. I: comparison of placebo and single dose of human synthetic secretin. Arch Dis Child 2003;88:731-736.

10. Rawlings ND, Barrett AJ. Families of cysteine peptidases. Methods Enzymol 1994;244:461-486.

11. Adams JB. Summary of dietary, nutritional, and medical treatments for autism based on over 150 published research studies. ARI Publication 40. 2013 [cited 2014 Dec 1]. Available from: http://autism.asu.edu.

12. Buie T, Campbell DB, Fuchs GJ 3rd, Furuta GT, Levy J, Vandewater J, et al. Evaluation, diagnosis, and treatment of gastrointestinal disorders in individuals with ASDs: a consensus report. Pediatrics 2010;125 Suppl 1:S1-S18.

13. Horvath K, Papadimitriou JC, Rabsztyn A, Drachenberg C, Tildon JT. Gastrointestinal abnormalities in children with autistic disorder. J Pediatr 1999;135:559-563.

14. Williams BL, Hornig M, Buie T, Bauman ML, Cho Paik M, Wick I, et al. Impaired carbohydrate digestion and transport and mucosal dysbiosis in the intestines of children with autism and gastrointestinal disturbances. PLoS One 2011;6:e24585.

15. Kushak RI, Lauwers GY, Winter HS, Buie TM. Intestinal disaccharidase activity in patients with autism: effect of age, gender, and intestinal inflammation. Autism 2011;15:285-294. 